\title{
Bebidas azucaradas: la batalla contra el sobrepeso y la obesidad en México
}

\author{
Lizbeth de la Cruz y Julieta Garduño
}

\begin{abstract}
Resumen
Un porcentaje importante de adultos mexicanos están ganando peso sin control, además, los datos de las encuestas nacionales de salud revelan que los niños también son víctimas de una excesiva acumulación de grasa en sus cuerpos. Lo anterior tiene sentido, ya que los adultos son los encargados de la alimentación de los niños, y si ellos mismos no se procuran una alimentación y un estilo de vida saludables, no pueden ofrecerlos a los menores. Tener sociedades con sobrepeso y obesidad es equivalente a tener sociedades enfermas, los riesgos en la salud van desde problemas psicológicos hasta problemas cardiovasculares y/o el desarrollo de alguna discapacidad, con posibles repercusiones económicas. Para tratar de frenar este incremento de peso en nuestra población, se desarrollaron diversas políticas públicas: una de ellas se implementó en el año 2014 y consistió en gravar a las bebidas azucaradas con un impuesto. En este artículo se revisaron algunos de los cambios que ocasionó esta propuesta en la población mexicana, se descubrió que, a seis años de su implementación, el consumo de refrescos ha disminuido considerablemente y, además, diversos modelos matemáticos prevén que este hecho ayudará a disminuir el porcentaje de personas con sobrepeso u obesidad en los próximos años, una proyección alentadora.
\end{abstract}

Palabras clave: reducción en el consumo, incremento de precio, bebidas gravadas, azúcar añadido, prevención

\section{SUGAR-SWEETENED BEVERAGES: THE BATTLE AGAINST OVERWEIGHT AND OBESITY IN MEXICO}

\begin{abstract}
A large percentage of Mexican adults are gaining weight uncontrollably. Data from national health surveys reveal that children are also becoming victims of this. This makes a great deal of sense as adults are responsible for feeding their children; if they are incapable of following a balanced diet and lifestyle, their children will unlikely develop healthy habits. High levels of overweight and obesity in populations lead to sick societies. Furthermore, health risks caused by obesity varying from psychological, cardiovascular, and other disability problems may lead to serious economic repercussions in these societies. To stop weight gain in Mexican population, various public policies have been developed. One such policy is the 2014 taxing of sugar-sweetened beverages with the intention to reduce their consumption. This article summarizes results associated with the sugar sweetened beverage tax and a reduction in soft drinks consumption in the Mexican population. Diverse mathematical models suggest a likelihood that the percentage of obesity and overweight people will also follow a downward trend, a good first step in improving the quality of life in Mexico.
\end{abstract}

Keywords: sugar intake reduction, price increase, taxed beverages, added sugar, prevention.

Recepción: 15/07/2020. Aprobación: 22/02/2021. Dol: http://doi.org/10.22201/cuaieed.16076079e.2021.22.3.6 


\section{Lizbeth de la Cruz}

ddlc@uw.edu

Estudió la Licenciatura en Investigación Biomédica Básica y el Doctorado en Ciencias Biomédicas, en la Facultad de Medicina de la Universidad Nacional Autónoma de México (UnAm). Fue profesora de la asignatura de Fisiología en la Facultad de Medicina de la unAm de 2012 a 2017. Realizó una estancia postdoctoral en el Centro de Ciencias de la Complejidad (C3) de la unAm, y actualmente se encuentra realizando su segunda estancia postdoctoral en la Universidad de Washington. Su campo de investigación se ha enfocado en la regulación de canales iónicos y la excitabilidad de neuronas y células beta pancreáticas por lípidos de membrana. Adicionalmente, colabora con grupos multidisciplinarios del C3 para entender la complejidad de la obesidad y diversas enfermedades metabólicas, como la diabetes, en la población mexicana.

\section{Julieta Garduño}

julietagt@unam.mx

Estudió Biología en la Universidad Autónoma Metropolitana, Xochimilco, y realizó su maestría y doctorado en el departamento de Fisiología, Biofísica y Neurociencias del Centro de Investigación y de Estudios Avanzados del IPN (Cinvestav), enfocándose en el estudio de un sistema neurosecretor de crustáceos, análogo al eje hipotálamo-neurohipófisis de los mamíferos. Realizó una estancia posdoctoral en el Instituto Cajal en Madrid, España. Durante esos años estuvo trabajando en el entendimiento de los mecanismos que regulan la excitabilidad de las neuronas hipocampales. Desde 2005 se encuentra trabajando en el Departamento de Fisiología de la Facultad de Medicina de la UNAM, en donde actualmente es profesora de tiempo completo, y donde imparte la asignatura de Fisiología y desarrolla líneas de investigación que involucran el estudio de los mecanismos sinápticos y extrasinápticos que regulan la actividad eléctrica en áreas hipotalámicas involucradas en la regulación de la ingesta de alimentos y el gasto energético. 


\section{Introducción}

Desafortunadamente, México ocupa el primer lugar a nivel mundial en la prevalencia de obesidad infantil (5 a 11 años) y el segundo en adultos mayores de 20 años (ENSANUT, 2016; UNICEF, 2019). Estos son datos muy preocupantes, pues engloban una problemática mayúscula, ya que el sobrepeso y la obesidad son factores de riesgo que pueden desencadenar enfermedades crónicas como las afecciones cardiovasculares y la diabetes. Recientemente, la obesidad también resultó ser un detonante de complicaciones para los pacientes con covid-19, lo cual demostró la importancia de este padecimiento en la salud humana (Stefan et al., 2021).

En las últimas cuatro décadas, los problemas de obesidad y sobrepeso en todas las edades comenzaron a incrementar de manera llamativa y preocupante a nivel mundial. Específicamente, en México, entre los años 1988 y 2018, la prevalencia de sobrepeso y obesidad en adultos aumentó de 34.5\% a 75.2\%. En el caso de los niños en edad escolar (de 5 a 11 años) el incremento fue de 8.8\% (pasó de 26.9\% a 35.7\%). En los adolescentes (de 12 a 19 años), en el 2012, el sobrepeso y la obesidad eran de 34.9\% y en el año 2018 la prevalencia combinada se elevó a 41.5\% (ENSANUt, 2014, 2018). Gracias a estos datos se puede notar cómo 3 de cada 10 menores, 4 de cada 10 adolescentes y 7 de cada 10 adultos padecen sobrepeso u obesidad; y éstas son cifras alarmantes.

Del análisis de los datos anteriores, surgen muchísimos cuestionamientos, todos encaminados a reflexionar y a trabajar en torno a qué hacemos o qué debemos evitar hacer para que nuestra población no gane peso de manera desenfrenada y sin control. En relación con la primera pregunta en este artículo analizamos si la estrategia gubernamental de gravar a las bebidas azucaradas con un impuesto impactó o no en su consumo. También, discutimos si dicha estrategia puede prevenir más casos de sobrepeso y de obesidad en nuestra población. Con respecto a lo que debemos evitar hacer, aquí planteamos la relevancia que tiene moderar el consumo de bebidas azucaradas, tanto en la prevención del desarrollo de sobrepeso y de obesidad, como en el esfuerzo de las personas que buscan volver a su peso ideal o desean mantener un peso saludable.

\section{Sobrepeso y obesidad ¿cuál es la causa?}

Primero distingamos la diferencia entre estos dos padecimientos: la Organización Mundial de la Salud (oms) define al sobrepeso y a la obesidad como una acumulación anormal y excesiva de grasa que puede ser perjudicial para la salud. Frecuentemente, se utiliza el índice de masa corporal (IMC) como un indicador simple de la relación entre el peso y la talla para identificar si un adulto padece de sobrepeso o de obesidad. Este se calcula dividiendo el peso de la persona, expresado en kilogramos, entre el cuadrado de su talla en metros $\left(\mathrm{kg} / \mathrm{m}^{2}\right)$. Así, la oms define a una persona adulta con sobrepeso, si su Imc es igual o mayor a 25 y a una persona adulta con obesidad si dicho índice es igual o mayor a 30. 
Actualmente, sabemos que el sobrepeso y la obesidad son enfermedades multifactoriales, es decir, más de una variable es responsable de su desarrollo (ver figura 1). Por ejemplo, pueden ser causadas por la carga genética, los malos hábitos de alimentación y/o la vida sedentaria. Aunque diversas investigaciones, como las realizadas en el Instituto Nacional de Medicina Genómica (INMEGEN) por el grupo de Canizales Quinteros, sugieren que la carga genética del mexicano promueve el desarrollo de la obesidad (León-Mimila et al., 2013; Villamil-Ramírez et al., 2017). Considerando que esta pandemia es un producto de casi cuatro décadas, limitarse a la carga genética como la única explicación, no parece factible. Alternativamente, diversas investigaciones han reportado que el consumo excesivo de carbohidratos, principalmente de "azúcares añadidos", por parte de la población, es un factor clave para el desarrollo de esta enfermedad.

Figura 1. Factores involucrados en el sobrepeso y la obesidad. Elaboración: Roselia Garduño Torres.

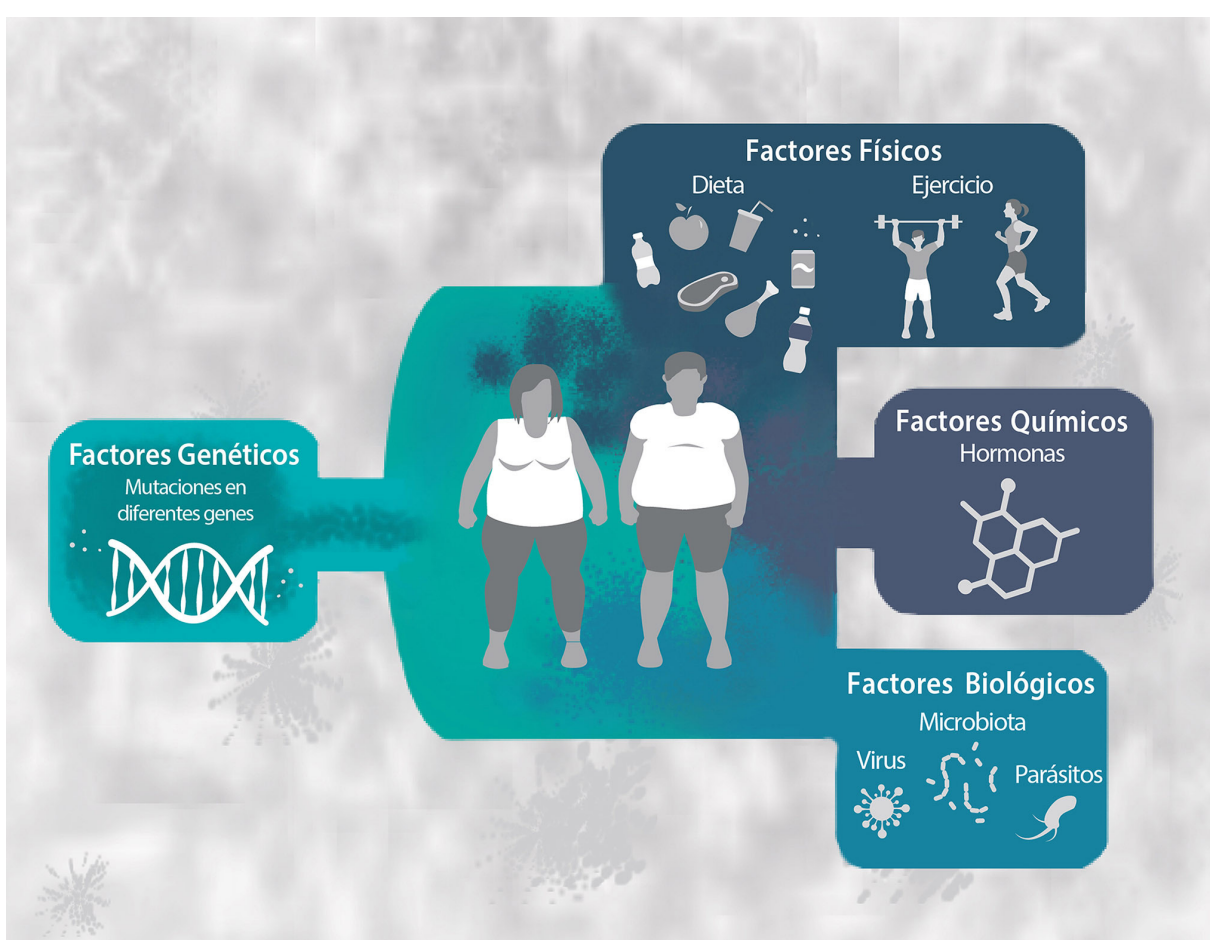

Los azúcares añadidos, cuyo origen se encuentra, por ejemplo, en el azúcar de mesa, deben diferenciarse de los azúcares intrínsecos de frutas y verduras frescas. Es interesante señalar que las bebidas azucaradas son la principal fuente de azúcares añadidos dentro de la población mexicana (Sánchez-Pimienta et al., 2016). Cada mexicano consume, aproximadamente, 160 litros de refresco al año, mientras que el promedio mundial es de 77 litros (López Munguía, 2007, ver figura 2). En promedio, se estima que cada persona ingiere 238 calorías al día únicamente por el consumo de estas bebidas. Estos datos muestran cómo el consumo de azúcares añadidos de los mexicanos sobrepasa, por mucho, la ingesta diaria recomendada por la oms, que es menos de 10\% de las necesidades energéticas diarias, y coloca una alerta roja sobre nuestro país y sobre qué podemos hacer para disminuir la incidencia de la obesidad. 
Figura 2. Consumo promedio anual de refresco del mexicano comparado con el promedio mundial. Elaboración: Roselia Garduño Torres.

\section{Cantidad de refresco al año}
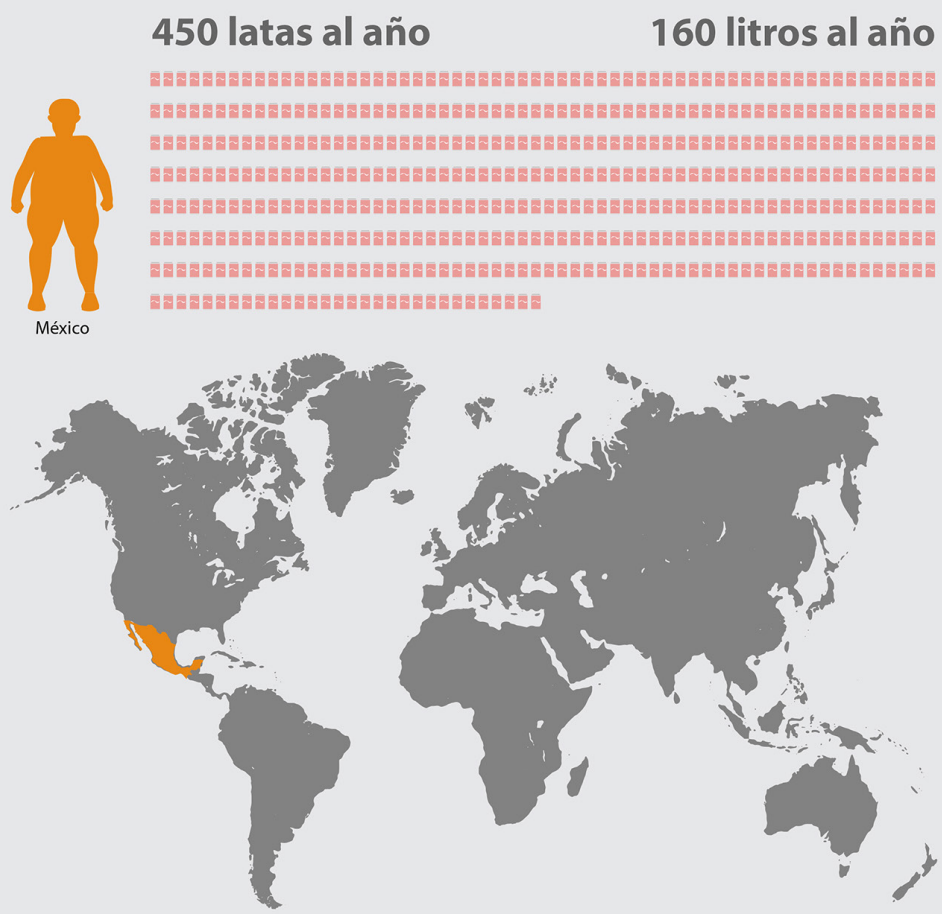

217 latas al año

77 litros al año

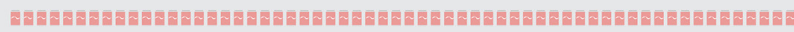

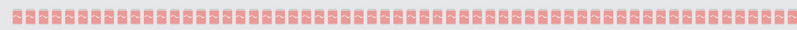

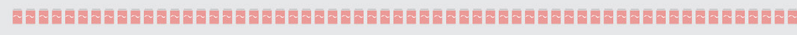

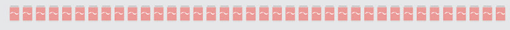

$355 \mathrm{ml}$.

\section{Dimensionemos el problema con un ejemplo, ¿te suena familiar el caso de este adolescente?}

Juan es un adolescente de 17 años que no realiza ningún deporte regularmente. Basados en su edad, género y actividad física, él deberá de consumir 2000 calorías diarias. Con esto en mente, supongamos que en un día ordinario Juan ingiere un néctar de mango de $500 \mathrm{ml}$ durante el receso de la jornada escolar y que, aparte, en su casa decide beber un refresco de cola de $600 \mathrm{ml}$ para acompañar su comida, este producto le aportó 252 calorías al joven, mientras que el néctar de fruta le sumó otras 305 calorías a su ingesta diaria. Al sumar las cantidades de ambas bebidas azucaradas podemos notar que Juan consumió un total de 557 calorías, lo que cubre 27.85\% de sus necesidades energéticas diarias. Mientras que, como ya mencionamos, los azúcares añadidos deberían de aportarle menos de 10\% (recomendación de la oms). 
Claramente, Juan consumió casi 18\% más de azúcares añadidos en este día hipotético y si esta conducta se arraiga como una costumbre, su ingesta recomendada será rebasada diariamente y aún sin tomar en cuenta otras posibles fuentes de azúcar que podría tener en su dieta (pastelillos, pan dulce y tamales, por mencionar algunos ejemplos). Los azúcares añadidos que Juan estaría consumiendo al día le estarían aportando más calorías de las que él necesita y de las que él podría gastar al realizar sus actividades cotidianas. Los adipocitos del cuerpo de Juan, que son las células que conforman al tejido graso, guardarán el exceso de carbohidratos en forma de grasa y sin duda alguna, él engordará.

De manera sumamente interesante sabemos que el resultado de ingerir tan sólo 20 calorías de más, lo equivalente a una cucharadita de azúcar, cada día durante un año, implica un kilogramo más de peso. Quizá suenen muy poquitas calorías, pero en 5 años, si Juan continúa con malos hábitos alimenticios y con una escasa actividad física ganará, por lo menos, 5 kilogramos de peso (esto si suponemos que su exceso de ingesta de carbohidratos es de únicamente 20 calorías más por día, aunque en la situación ejemplificada, Juan ingirió 357 calorías extra, ver figura 3).

Figura 3. El resultado de ingerir un excedente equivalente a una cucharadita de azúcar cada día implicaría al año un kilogramo más de peso. Elaboración: Roselia Garduño Torres.

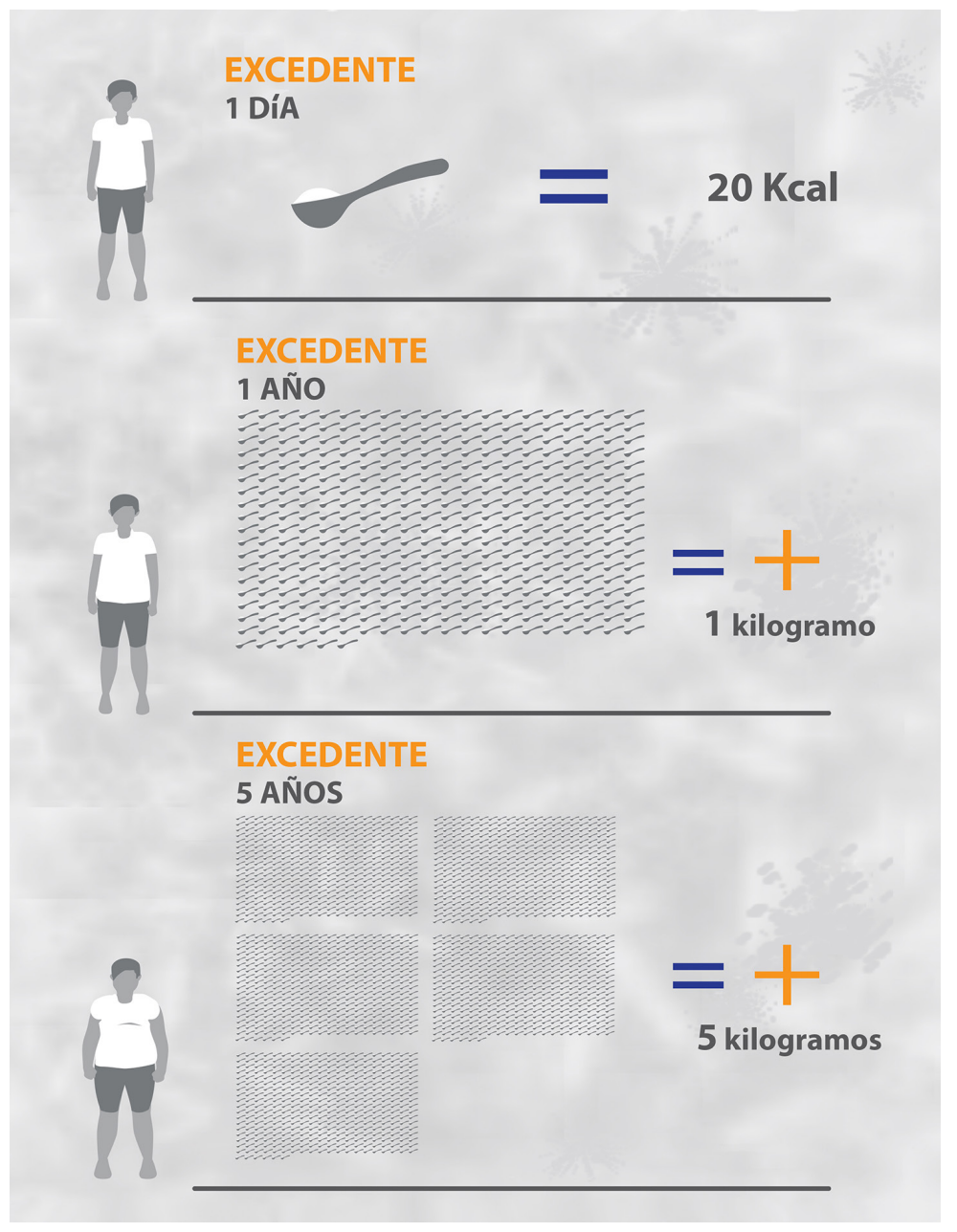


En este punto, nos podemos preguntar si la actitud y el estilo de vida de Juan tendrían alguna repercusión futura en nuestro Sistema Nacional de Salud y la respuesta es un sí, pues este joven es un candidato potencial para desarrollar afecciones cardiovasculares y trastornos metabólicos (sobrepeso, obesidad, trigliceridemia, intolerancia a la glucosa, etc.) que podrían detonar en diabetes. Hay que considerar que el costo anual de su enfermedad puede ir desde los nueve mil hasta los treinta y siete mil pesos, esto depende del número de fármacos que necesite para tener controlado su padecimiento y dicho costo podría incrementarse de manera alarmante si la enfermedad de Juan se cursa con complicaciones como la falla renal, la pérdida de la visión o la infección de una extremidad que podría terminar en la amputación del miembro. En cualquiera de los casos, la calidad de vida del paciente quedará seriamente mermada y estos padecimientos podrían afectar su autosuficiencia y su productividad.

En consecuencia, habrá un gasto importante tanto para el Sistema Nacional de Salud como para Juan y su familia. Y si además se toma en consideración que podría desarrollar la diabetes a una edad temprana, el panorama se torna aún más sombrío ya que actualmente, en México, 13\% de adultos tiene esta enfermedad. Así pues, si la historia de Juan se repite, por ejemplo, en 24 millones de personas (20\% de la población mexicana), el desenlace será un sistema de salud con una capacidad rebasada, perpetuando la pobreza y la atención deficiente de los pacientes.

\section{El impuesto a las bebidas con azúcar añadido}

El gobierno de nuestro país ha intentado frenar la epidemia de sobrepeso y de obesidad mediante diversas políticas públicas, por ejemplo, se incrementaron los precios de alimentos potencialmente no saludables por medio de un impuesto y los alimentos con alta densidad calórica (100 gramos de producto con más de 275 calorías) se gravan con 8\%, lo cual incluye botanas, confitería, chocolates, postres y dulces, mantequilla de cacahuate, helados y nieves. Asimismo, desde el año 2014, los mexicanos pagamos un peso más cada vez que compramos alguna bebida saborizada con azúcar añadido. Cabe destacar que un porcentaje de la recaudación del impuesto a las bebidas azucaradas se destina a programas de prevención y de control del sobrepeso y de la obesidad, así como a programas que ayudan a proveer del acceso al agua potable a las comunidades que carecen de ella.

\section{¿Cuáles han sido los resultados tras la implementación de este impuesto?}

Después de 6 años del implemento de este impuesto a las bebidas azucaradas como política de salud para disminuir su consumo, diversos trabajos se han enfocado a evaluar los resultados de esta medida. Tras un año, Colchero y colaboradores (2016) reportaron que la compra de estas bebidas disminuyó 
en promedio 6\% y que se incrementó la adquisición de bebidas sin impuesto, como el agua embotellada. Asimismo, los autores mostraron que se redujo la adquisición de bebidas saborizadas en mayor escala en la población con menor ingreso económico, sector más afectado por el problema de la obesidad y el sobrepeso. Después del segundo año de la aplicación del impuesto, también se reportó una disminución en la compra de bebidas azucaradas, para este caso, de 7.3\% (Colchero et al., 2017). Asimismo, otro grupo de investigación encontró que si las personas tienen el conocimiento de la aplicación del impuesto sobre las bebidas azucaradas, las hace más susceptibles a reducir su consumo en 30\% (Álvarez-Sánchez et al., 2018). De manera muy interesante, los resultados publicados por Shu Wen Ng et al., (2018) demostraron que en los hogares con el consumo más alto de bebidas saborizadas se presentaron las mayores reducciones de consumo (17.5\%), lo cual logró incrementar la obtención de bebidas sin impuesto en $12 \%$.

Finalmente, Fernández y Raine (2019) indicaron que aplicar el impuesto sobre las bebidas saborizadas sí modifica los patrones de consumo, aunque esta medida debe ser complementada con otras intervenciones como incrementar el acceso a bebidas no endulzadas y educar acerca del consumo de bebidas saludables (ver figura 4). De igual manera, puntualizan en la importancia de trabajar de la mano con la industria refresquera para la reformulación de productos. Así, es importante destacar que esta política de salud proporciona buenos resultados en el país y que, además, sirve como base para que otros países tomen acciones directas para reducir su consumo de bebidas azucaradas.

Figura 4. Consecuencias del impuesto sobre las bebidas saborizadas en los patrones de consumo. Elaboración: Roselia Garduño Torres..
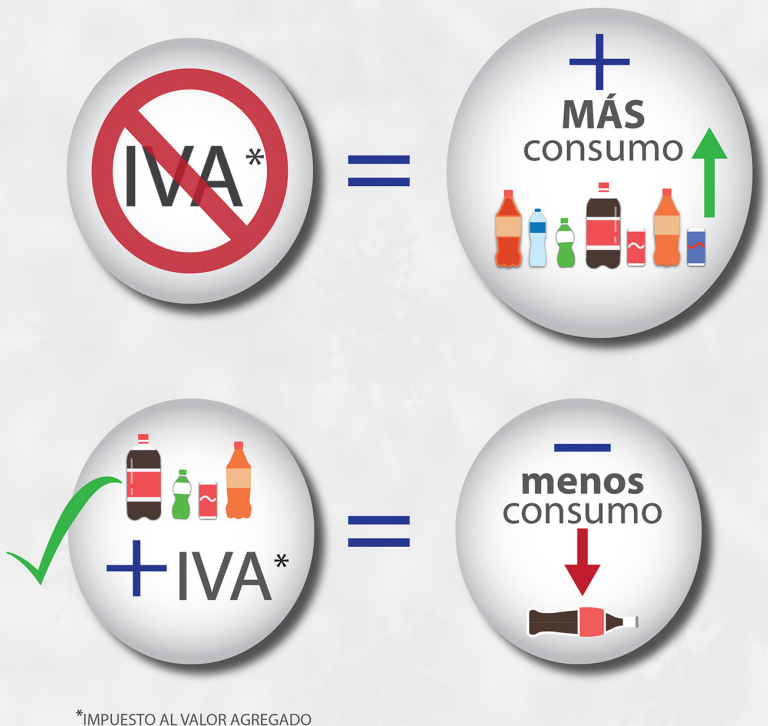

*IMPUESTO AL VALOR AGREGADO

También, es necesario puntualizar que si bien, ya se logró reducir el consumo de bebidas con azúcar añadido, la encuesta de ENSANUT 2018, mostró que estas 
"Bebidas azucaradas: la batalla contra el sobrepeso y la obesidad en México"

Lizbeth de la Cruz y Julieta Garduño

Vol. 22, Núm. 3, mayo-junio 2021

Revista Digital Universitaria

bebidas siguen aportando más calorías de las recomendadas en los adultos, adolescentes y niños (Rodríguez-Ramírez et al., 2020). Esto demuestra que aún prevalece una urgencia por continuar con la implementación de nuevas estrategias para la prevención y la reducción de los índices de sobrepeso y obesidad en la población mexicana.

Finalmente, aunque aún es muy temprano para observar los resultados del impuesto de las bebidas azucaradas sobre los índices de sobrepeso u obesidad directamente, diferentes predicciones, basadas en modelos matemáticos, estiman que los impuestos a las bebidas azucaradas podrían reducir su consumo de 34 a 47 calorías por día en adultos y de 40 a 51 en niños, lo cual, como consecuencia, resulta en una disminución de $1.7 \mathrm{~kg}$ hasta $2 \mathrm{~kg}$ por año (Lin et al., 2011). Asimismo, se pronostica que se podrían prevenir de 40 a 78 nuevos casos de obesidad cada año (Smith et al., 2010; Goiana-da-Silva et al., 2020).

\section{Conclusiones}

Es de aplaudir que nuestro país haya iniciado una acción concreta para buscar una reducción del sobrepeso y de la obesidad, enfermedades que tanto aquejan a la población. Por ahora, lo que nos queda, es observar cómo avanza esta estrategia y esperar a que vengan pronto los resultados positivos. Eso sí, debemos considerar a esta estrategia como una de las múltiples propuestas que se deben generar para tener un verdadero impacto a corto plazo, especialmente en nuestras nuevas generaciones.

El año 2020 nos recordó la urgencia de promover e implementar programas para mejorar la salud metabólica de la población mexicana, y así, lograr vencer a las dos grandes pandemias mundiales: la de la covid-19 y la de la obesidad.

\section{Referencias}

* Álvarez-Sánchez, C., Contento, I., Jiménez-Aguilar, A., Koch, P., Gray, H. L., Guerra, L. A., Rivera-Dommarco, J., Uribe-Carvajal, R. y Shamah-Levy, T. (2018). Does the Mexican sugar-sweetened beverage tax have a signaling effect? ENSANUT 2016. PloS one, 13(8), e0199337. https://doi.org/10.1371/journal.pone.0199337

* Colchero, M. A., Popkin, B. M., Rivera, J. A. y Ng, S. W. (2016). Beverage purchases from stores in Mexico under the excise tax on sugar sweetened beverages: observational study. BMJ, 352, h6704. https://doi.org/10.1136/bmj.h6704

* Colchero, M. A., Rivera-Dommarco, J., Popkin, B. M. y Ng, S. W. (2017). In Mexico, Evidence Of Sustained Consumer Response Two Years After Implementing A Sugar-Sweetened Beverage Tax. Health affairs, 36(3), 564-571. https://doi. org/10.1377/hlthaff.2016.1231

* Encuesta Nacional de Salud y Nutrición. (2014, 2016, 2018). Instituto Nacional de Salud Pública, Secretaría de Salud, Gobierno de México. https://ensanut.insp.mx/ 
"Bebidas azucaradas: la batalla contra el sobrepeso y la obesidad en México"

Lizbeth de la Cruz y Julieta Garduño

Vol. 22, Núm. 3, mayo-junio 2021

Revista Digital Universitaria

* Fernandez, M. A. y Raine, K. D. (2019). Insights on the Influence of Sugar Taxes on Obesity Prevention Efforts. Current Nutrition Reports, 8(4), 333-339. https://doi. org/10.1007/s13668-019-00282-4

* Fondo de las Naciones Unidas para la Infancia (unicef). Estado Mundial de la Infancia 2019. Niños, alimentos y nutrición. Crecer bien en un mondo en transformación. https://www.unicef.org/es/informes/estado-mundial-de-la-infancia-2019

* Goiana-da-Silva, F., Severo, M., Cruz E Silva, D., Gregório, M. J., Allen, L. N., Muc, M., Morais Nunes, A., Torres, D., Miraldo, M., Ashrafian, H., Rito, A., Wickramasinghe, K., Breda, J., Darzi, A., Araújo F. y Lopes, C. (2020). Projected Impact of the Portuguese Sugar-Sweetened Beverage Tax on Obesity Incidence Across Different Age Groups: A Modelling Study. PLoS Med, 17(3), e1003036. https://doi.org/10.1371/ journal.pmed.1003036

- León-Mimila, P., Villamil-Ramírez, H., Villalobos-Comparán, M., Villarreal-Molina, T., Romero-Hidalgo, S., López-Contreras, B., Gutiérrez-Vidal, R., Vega-Badillo, J., Jacobo-Albavera, L., Posadas-Romeros, C., Canizalez-Román, A., Río-Navarro, B. D., Campos-Pérez, F., Acuña-Alonzo, V., Aguilar-Salinas, C., y Canizales-Quinteros, S. (2013). Contribution of common genetic variants to obesity and obesityrelated traits in mexican children and adults. PloS one, 8(8), e70640. https://doi. org/10.1371/journal.pone.0070640

* Lin, B. H., Smith T. A., Lee J. Y. y Hall, K.D. (2011). Measuring weight outcomes for obesity intervention strategies: The case of a sugar-sweetened beverage tax. Econ Hum Biol, 9(4), 329-41. https://doi.org/10.1016/j.ehb.2011.08.007

* López Munguía, A. (2007). Azúcar: hechos y mitos. ¿Cómo ves?,113. http://www. comoves.unam.mx/numeros/articulo/113/azucar-hechos-y-mitos

* Ng, S. W., Rivera, J. A., Popkin, B. M. y Colchero, M. A. (2018). Did high sugarsweetened beverage purchasers respond differently to the excise tax on sugarsweetened beverages in Mexico? Public health nutrition, 22(4), 750-756, 1-7. https:// doi.org/10.1017/S136898001800321X

* Rodríguez-Ramírez, S., Gaona-Pineda, E. B., Martínez-Tapia, B., Arango-Angarita, A., Kim-Herrera, E.Y., Valdez-Sánchez, A., Medina-Zacarías, M.C., Ramírez-Silva, I. y Shamah-Levy, T. (2020, noviembre-diciembre). Consumo de grupos de alimentos y su asociación con características sociodemográficas en población mexicana. Ensanut 2018-19. Salud Publica Mex, 62(6), 693-703. https://saludpublica.mx/ index.php/spm/article/view/11529

* Sánchez-Pimienta, T. G., Batis, C., Lutter, C. K. y Rivera, J. A. (2016). SugarSweetened Beverages Are the Main Sources of Added Sugar Intake in the Mexican Population. The Journal of nutrition, 146(9), 1888S-1896S. https://doi.org/10.3945/ jn.115.220301

Smith, T. A., Biing-Hwan, L.y Jonq-Ying, L. (2010). Taxing Caloric Sweetened Beverages: Potential Effects on Beverage Consumption, Calorie Intake, and Obesity. ERR-100, U.S. Department of Agriculture, Economic Research Service. https://cutt.ly/ZcASaGK

* Stephan, N., Birkenfeld, A. L. y Schulze, M. B. (2021). Global pandemics interconnected-obesity, impaired metabolic health and covid-19. Nature Reviews in Endocrinology, 17, 135-149. https://doi.org/10.1038/s41574-020-00462-1 
* Villamil-Ramírez, H., León-Mimila, P., Macias-Kauffer, L. R., Canizalez-Román, A., Villalobos-Comparán, M., León-Sicairos, N., Vega-Badillo, J., Sánchez-Muñoz, F., López-Contreras, B., Morán-Ramos, S., Villarreal-Molina, T., Zurita, L. C., CamposPérez, F., Huertas-Vazquez, A., Bojalil, R., Romero-Hidalgo, S., Aguilar-Salinas, C. A. y Canizales-Quinteros, S. (2017). A combined linkage and association strategy identifies a variant near the GSTP1 gene associated with BMI in the Mexican population. Journal of human genetics, 62, 413-418. https://doi.org/10.1038/ jhg.2016.145

* Wickramasinghe, K., Breda, J., Darzi, A., Araújo, F., Lopes, C. (2020). Projected impact of the Portuguese sugar-sweetened beverage tax on obesity incidence across different age groups: A modelling study. PLoS medicine, 17(3), e1003036. https://doi.org/10.1371/journal.pmed.1003036

\section{Cómo CITAR ESTE ARTículo}

* De la Cruz, Lizbeth y Garduño, Julieta. (2021, mayo-junio). Bebidas azucaradas: la batalla contra el sobrepeso y la obesidad en México. Revista Digital Universitaria (RDU), 22(3). Dol: http://doi.org/10.22201/cuaieed.16076079e.2021.22.3.6 